\title{
Interplay of dynamical and explicit chiral symmetry breaking effects on a quark
}

\author{
Fernando E. Serna, ${ }^{1,2,3, *}$ Chen Chen, ${ }^{2, \dagger}$ and Bruno El-Bennich ${ }^{2,3, \$}$ \\ ${ }^{1}$ Instituto Tecnológico de Aeronáutica, DCTA, 12228-900 São José dos Campos, SP, Brazil \\ ${ }^{2}$ Instituto de Física Teórica, Universidade Estadual Paulista, Rua Dr. Bento Teobaldo Ferraz, \\ 271-Bloco II, 01140-070 São Paulo, SP, Brazil \\ ${ }^{3}$ Laboratório de Física Teórica e Computacional, Universidade Cruzeiro do Sul, \\ Rua Galvão Bueno, 868, 01506-000 São Paulo, São Paulo, Brazil
}

(Received 17 December 2018; published 20 May 2019)

\begin{abstract}
The relative contributions of explicit and dynamical chiral symmetry breaking in QCD models of the quark-gap equation are studied in dependence of frequently employed Ansätze for the dressed interaction and quark-gluon vertex. The explicit symmetry breaking contributions are defined by a constituent-quark sigma term whereas the combined effects of explicit and dynamical symmetry breaking are described by a Euclidean constituent-mass solution. We extend this study of the gap equation to a quark-gluon vertex beyond the Abelian approximation complemented with numerical gluon- and ghost-dressing functions from lattice QCD. We find that the ratio of the sigma term over the Euclidean mass is largely independent of nonperturbative interaction and vertex models for current-quark masses, $m_{u, d}(\mu) \leq m(\mu) \leq m_{b}(\mu)$, and equal contributions of explicit and dynamical chiral symmetry breaking occur at $m(\mu) \approx 400 \mathrm{MeV}$. For massive solutions of the gap equation with lattice propagators this value decreases to about $220 \mathrm{MeV}$.
\end{abstract}

DOI: 10.1103/PhysRevD.99.094027

\section{INTRODUCTION}

Strong interactions are singularly characterized by a most effective mass-generating mechanism driven by dynamical chiral symmetry breaking (DCSB). The scope and magnitude of the mass generation are unlike that observed in quantum electrodynamics, for example, and the origin of this chiral symmetry breaking is thought to be intimately related with confinement [1]. Indeed, the emergence of a constituent-quark mass scale and the fact that DCSB contributes to nearly $98 \%$ of visible mass has become a paradigm in contemporary hadron physics $[2,3]$.

The impact of DCSB is evident in the light sector and plays an eminent role in describing why the nucleon's mass is about 2 orders of magnitude larger than that of its three bare constituents. For heavier quarks, starting with the strange quark, the effect of DCSB is gradually attenuated and the $b$-quark's constituent mass is almost completely due to the Higgs mechanism [4-7].

\footnotetext{
*fernando.enrique@unesp.br

†chenchen@ift.unesp.br

•bruno.bennich@cruzeirodosul.edu.br
}

Published by the American Physical Society under the terms of the Creative Commons Attribution 4.0 International license. Further distribution of this work must maintain attribution to the author(s) and the published article's title, journal citation, and DOI. Funded by SCOAP ${ }^{3}$.
As a suitable measure for the effect of DCSB one can use the dimensionless ratio $\sigma_{f} / M_{f}^{E}$ [5], where $\sigma_{f}$ is the constituent quark's sigma term and $M_{f}^{E}$ is a Euclidean constituent-quark mass. This ratio quantifies the contribution of explicit chiral symmetry breaking (CSB) to the dressed quark-mass function compared with the sum of both CSB and DCSB. It turns out that somewhere between the strange- and charm-quark mass the effects of CSB and DCSB are of the same order [5,7]. Moreover, while the weak decay constants of light pseudoscalar and vector mesons increase with the light current-quark mass, they level off somewhere between the strange- and charm-quark mass and fall off for heavier quark masses as $f_{M}=$ $1 / \sqrt{ } M_{M}[1,8]$. On the other hand, the weak decay constants of radially excited quarkonia can be shown to vanish in the chiral limit but, though suppressed, their values increase again in a mass range somewhere between the $\bar{s} s$ and $\bar{c} c$ quarkonia [9-11].

Clearly, dynamical effects on the dressed-mass function in the intermediate range between these two current-quark mass scales have a substantial impact on hadronic observables; an analogue observation is that $\mathrm{SU}(4)_{F}$ flavor symmetry is badly broken compared with $\mathrm{SU}(3)_{F}[12-14]$.

In continuum approaches to quantum chromodynamics (QCD), such as the Dyson-Schwinger equation (DSE) for the quark, the strength of DCSB is governed by two ingredients in its integral kernel: the gluon-dressing function [15-17] and the dressed quark-gluon vertex [18-36]. 
Failure to produce sufficient support results in a Wigner solution of the gap equation, and thus any symmetrypreserving truncation must compensate for lacking interaction strength [37]. The question arises how the pattern of DCSB and the relative effects between CSB and DCSB, for a given flavor, depend on the simplifications applied to these kernels. It turns out that the contributions of CSB and DCSB to the constituent-quark mass are approximately similar halfway between the strange and charm currentquark masses in the leading symmetry-preserving truncation of the quark's DSE and given functional form of the model interaction, namely the Maris-Tandy (MT) model [37].

Including additional tensor structures of the dressed quark-gluon vertex is commonly compensated by readjusting the infrared-interaction strength of this model "dressing" function; the functional form of the quark-mass function as well as the position of associated complexconjugate mass poles are consequently modified [38]. While additional transverse vertex structures can be included in the DSE kernel without notable computational efforts, this is not the case for the integral kernel of antiquark-quark bound-state equations. Any BetheSalpeter kernel of the axial-vector vertex must satisfy an axial-vector Ward-Takahashi identity, and the truncation of the kernel must be consistent with that of the DSE. While this has been achieved to a certain degree (see the discussion in Ref. [1]), progress is still ongoing and necessary. We therefore limit ourselves to explore the effects of DCSB in truncations of the DSE with increasing complexity and associated quark- $\sigma$ terms and postpone more ambitious calculations of hadronic $\sigma$ terms to future studies.

We here contribute to gain additional insight in DCSB by studying the quark's DSE for different interactions and quark-gluon vertex Ansätze, including the MT model, its more recent modification which reflects the results of modern DSE and lattice studies on the gluon propagator [39], the leading rainbow truncation, the Ball-Chiu vertex and transverse tensor structures of the vertex. It is found that the behavior of the renormalization-point-invariant ratio $\sigma_{f} / M_{f}$ as a function of the current-quark mass is nearly independent of the integral kernel formed by the convolution of the vertex- and gluon-dressing functions. On the other hand, for a given flavor and interaction tuned to reproduce light-hadron observables, the Euclidean mass $M_{f}^{E}$ varies in a range of about $20 \%-30 \%$. The extension of this numerical study to a gap equation with gluon and ghost propagators obtained with lattice QCD simulations mirrors the findings with model interactions.

\section{QUARK DYSON-SCHWINGER EQUATION}

The DSEs are the quantum equations of motion of a field theory and can be derived starting from the path integral formalism. As such, they are described by an infinite tower

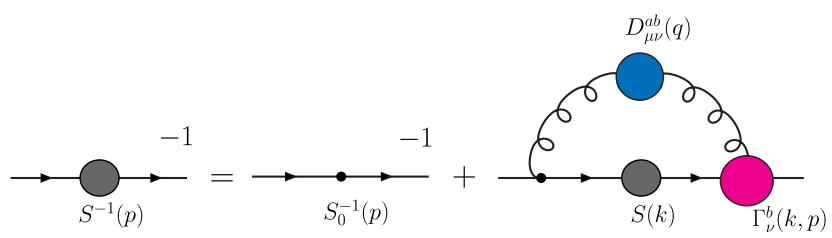

FIG. 1. Diagrammatic representation of the quark DysonSchwinger equation (1). Full circles denote fully dressed propagators and vertices.

of coupled exact integral equations. In QCD, the quark fields obey a DSE [1-3,40,41] for a given flavor, diagrammatically depicted in Fig. $1{ }^{1}$

$$
\begin{aligned}
S_{f}^{-1}(p)= & Z_{2}\left(i \gamma \cdot p+m_{f}^{\mathrm{bm}}\right) \\
& +Z_{1} g^{2} \int_{k}^{\Lambda} D_{\mu \nu}^{a b}(q) \frac{\lambda^{a}}{2} \gamma_{\mu} S_{f}(k) \Gamma_{\nu}^{b}(k, p),
\end{aligned}
$$

where $q=k-p, Z_{1}(\mu, \Lambda)$ and $Z_{2}(\mu, \Lambda)$ are the vertex and quark wave-function renormalization constants, respectively, and $\mu$ is the renormalization point. Infinite radiative gluon corrections yield the quark self-energy which modifies the current-quark bare mass $m_{f}^{\mathrm{bm}}(\Lambda)$, and where the integral is over the dressed gluon propagator, $D_{\mu \nu}(q)$, and the dressed quark-gluon vertex, $\Gamma_{\mu}^{a}(k, p)=\frac{1}{2} \lambda^{a} \Gamma_{\mu}(k, p)$; the color SU(3) matrices $\lambda^{a}$ are in the fundamental representation. In the integral, the abbreviation $\int_{k}^{\Lambda} \equiv \int^{\Lambda} d^{4} k /(2 \pi)^{4}$ represents a Poincare-invariant regularization with the regularization-mass scale $\Lambda$. We work in the Landau gauge, where the gluon propagator is purely transversal:

$$
D_{\mu \nu}^{a b}(q)=\delta^{a b}\left(\delta_{\mu \nu}-\frac{q_{\mu} q_{\nu}}{q^{2}}\right) \frac{\Delta\left(q^{2}\right)}{q^{2}},
$$

which defines the gluon-dressing function $\Delta\left(q^{2}\right)$.

The solutions to the gap equation (1) for spacelike momenta, $p^{2}>0$, can be decomposed into a vector and scalar piece,

$$
S_{f}(p)=\left[i \gamma \cdot p A_{f}\left(p^{2}\right)+\mathbb{I}_{D} B_{f}\left(p^{2}\right)\right]^{-1},
$$

and the renormalization condition

$$
Z_{f}\left(p^{2}\right)=1 /\left.A_{f}\left(p^{2}\right)\right|_{p^{2}=\mu^{2}}=1
$$

is imposed. Typically, in conjunction with the MT model discussed below, $A_{f}\left(p^{2}\right)$ is renormalized at a large spacelike momentum, $\mu=19 \mathrm{GeV} \gg \Lambda_{\mathrm{QCD}}$. More recent numerical results of the quark propagator's dressing

\footnotetext{
${ }^{1}$ We employ throughout a Euclidean metric in our notation: $\left\{\gamma_{\mu}, \gamma_{\nu}\right\}=2 \delta_{\mu \nu} ; \gamma_{\mu}^{\dagger}=\gamma_{\mu} ; \gamma_{5}=\gamma_{4} \gamma_{1} \gamma_{2} \gamma_{3}, \operatorname{tr}\left[\gamma_{4} \gamma_{\mu} \gamma_{\nu} \gamma_{\rho} \gamma_{\sigma}\right]=-4 \epsilon_{\mu \nu \rho \sigma}$; $\sigma_{\mu \nu}=(i / 2)\left[\gamma_{\mu}, \gamma_{\nu}\right] ; a \cdot b=\sum_{i=1}^{4} a_{i} b_{i}$; and $P_{\mu}$ timelike $\Rightarrow P^{2}<0$.
} 
functions from lattice QCD also allow for a much lower renormalization point $A_{f}(\mu \simeq 2 \mathrm{GeV})=1$. The mass function $M_{f}\left(p^{2}\right)=B_{f}\left(p^{2}, \mu^{2}\right) / A_{f}\left(p^{2}, \mu^{2}\right)$ is independent of $\mu$. The scalar function $B_{f}\left(p^{2}\right)$ is determined with another renormalization condition:

$$
\left.S_{f}^{-1}(p)\right|_{p^{2}=\mu^{2}}=i \gamma \cdot p+m_{f}(\mu) \mathbb{I}_{D},
$$

where $m_{f}(\mu)$ is the renormalized running quark mass:

$$
Z_{m}^{f}(\mu, \Lambda) m_{f}(\mu)=m_{f}^{\mathrm{bm}}(\Lambda) .
$$

Here, $Z_{m}^{f}(\mu, \Lambda)=Z_{4}^{f}(\mu, \Lambda) / Z_{2}^{f}(\mu, \Lambda)$ is the flavor-dependent mass-renormalization constant and $Z_{4}^{f}(\mu, \Lambda)$ is the renormalization constant associated with the Lagrangian's mass term; $Z_{2}$ and $Z_{4}$ are fixed by the renormalization conditions in Eqs. (4) and (5). Notably, $m_{f}(\mu)$ is the quarkmass function evaluated at a particular deep spacelike point, $p^{2}=\mu^{2}$, which makes contact with perturbative QCD:

$$
m_{f}(\mu)=M_{f}(\mu)
$$

Finally, we remark that the renormalization-group-invariant current-quark mass can be inferred from

$$
\hat{m}_{f}=\lim _{p^{2} \rightarrow \infty} M_{f}\left(p^{2}\right)\left[\frac{1}{2} \ln \left(\frac{p^{2}}{\Lambda_{\mathrm{QCD}}^{2}}\right)\right]^{\gamma_{m}},
$$

where $\gamma_{m}$ is the anomalous mass dimension.

\section{A. Quark-gluon vertex}

Due to asymptotic freedom, the behavior of the kernel at large momenta is known in perturbation theory in the domain $q^{2} \simeq p^{2} \simeq k^{2} \gtrsim 2 \mathrm{GeV}^{2}$ from which one can derive a sensible model for realistic DSE calculations [23] given by

$$
Z_{1} g^{2} D_{\mu \nu}(q) \Gamma_{\mu}(k, p)=Z_{2} q^{2} \mathcal{G}\left(q^{2}\right) D_{\mu \nu}^{\mathrm{free}}(q) \Gamma_{\mu}(k, p),
$$

where $D_{\mu \nu}^{\mathrm{free}}(q)=\left(\delta_{\mu \nu}-q_{\mu} q_{\nu} / q^{2}\right) / q^{2}$ is the free gluon propagator. In Eq. (9), the Abelianized Ward-GreenTakahashi identity (WGTI)

$$
q_{\mu} i \Gamma_{\mu}(k, p)=S^{-1}(k)-S^{-1}(p)
$$

has been enforced, $Z_{1}=Z_{2}$, which at one loop corresponds to neglecting the contributions of the three-gluon vertex to $\Gamma_{\mu}(k, p)$. Formally, this is equivalent to setting the renormalization constants for the ghost-gluon vertex and ghost wave function equal: $\tilde{Z}_{1}=\tilde{Z}_{3}$.
In the leading truncation we employ the Ansatz

$$
\Gamma_{\mu}(k, p)=Z_{2} \gamma_{\mu},
$$

where an additional factor $Z_{2}$ is included [42] to ensure multiplicative renormalizability of Eq. (1) and thus the renormalization-point independence of $M\left(p^{2}\right)$. When the Abelian approximation $Z_{1}=Z_{2}$ is used along with the rainbow-ladder (RL) truncation $Z_{1}=1$, it preserves the one-loop anomalous dimension of $M\left(p^{2}\right)$ [43]. Therefore, to make contact with early studies on the quark DSE, we also absorb the renormalization constants $Z_{2}^{2}$ from Eqs. (9) and (11) into the function $\mathcal{G}\left(q^{2}\right)$ in case of the MT model (18), and only in that case, which effectively describes the effects of both the gluon and the vertex dressing.

To go beyond this approximation, we treat the case of the Ball-Chiu (BC) Ansatz [33,34] which satisfies Eq. (10) by construction,

$$
\begin{aligned}
\Gamma_{\mu}^{\mathrm{BC}}(k, p)= & \Sigma_{A}\left(k^{2}, p^{2}\right) \gamma_{\mu}+\Delta_{A}\left(k^{2}, p^{2}\right) \gamma \cdot(k+p)(k+p)_{\mu} \\
& -i \Delta_{B}\left(k^{2}, p^{2}\right)(k+p)_{\mu} \mathbb{I}_{D},
\end{aligned}
$$

with the compact definitions:

$$
\begin{aligned}
& \Sigma_{\phi}\left(k^{2}, p^{2}\right)=\frac{\phi\left(k^{2}\right)+\phi\left(p^{2}\right)}{2}, \\
& \Delta_{\phi}\left(k^{2}, p^{2}\right)=\frac{\phi\left(k^{2}\right)-\phi\left(p^{2}\right)}{k^{2}-p^{2}},
\end{aligned}
$$

and $\phi\left(k^{2}\right)=A\left(k^{2}\right), B\left(k^{2}\right)$. The vertex in Eq. (12) clearly implies a flavor dependence via the vector and scalar functions $A_{f}\left(p^{2}\right)$ and $B_{f}\left(p^{2}\right)$.

The WGTI only constrains the Ball-Chiu components of $\Gamma_{\mu}(k, p)$ but extensive studies in perturbation theory have also shed light on the functional dependence of the transverse vertex components on $A\left(p^{2}\right)$ and $B\left(p^{2}\right)[30,31]$ in certain kinematic limits. We here use an Ansatz [44] which models the anomalous chromomagnetic moment:

$$
\begin{aligned}
\Gamma_{\mu}^{\mathrm{T}}(k, p)= & \frac{1}{2}\left[(k+p)_{\mu}^{\mathrm{T}} \gamma \cdot q+\gamma_{\mu}^{\mathrm{T}} \sigma_{\nu \rho}(k+p)_{\nu} q_{\rho}\right] \\
& \times \frac{\eta \Delta_{B}\left(k^{2}, p^{2}\right)}{\mathcal{M}\left(k^{2}, p^{2}\right)}+\eta \Delta_{B}\left(k^{2}, p^{2}\right) \sigma_{\mu \nu} q_{\nu},
\end{aligned}
$$

with $\ell_{\mu}^{T}:=T_{\mu \nu} \ell_{\nu}, T_{\mu \nu}=\delta_{\mu \nu}-q_{\mu} q_{\nu} / q^{2}, \quad \eta=0.325$ and introducing the function

$$
\mathcal{M}\left(k^{2}, p^{2}\right)=\frac{k^{2}+M^{2}\left(k^{2}\right)+p^{2}+M^{2}\left(p^{2}\right)}{2\left[M\left(k^{2}\right)+M\left(p^{2}\right)\right]} .
$$

Adding these transverse components to the Ball-Chiu vertex the nonperturbative quark-gluon vertex becomes the linear sum 


$$
\Gamma_{\mu}(k, p)=\Gamma_{\mu}^{\mathrm{BC}}(k, p)+\Gamma_{\mu}^{\mathrm{T}}(k, p) .
$$

Independent of perturbative results, the transverse dressing functions can be derived from Lorentz symmetries using a set of transverse WGTIs [45-47] and their functional form in the Abelian case can be found in Refs. [22,26]. In QCD, on the other hand, the fermiongauge vertex satisfies a Slavnov-Taylor identity (STI) $[48,49]$ which also leaves the transverse component undetermined. The studies of the Abelian transverse vertex identities were generalized to transverse STIs which lead to expressions that depend on the scalar- and vector-quark functions but also on the ghost-dressing function, the quark-ghost scattering amplitude and on a nontrivial, nonlocal four-point function which is a consequence of gauge invariance [50]. The latter term involves a Wilson line in QED and QCD and can be parametrized most generally by four tensor structures and corresponding form factors; similarly the most general quark-ghost scattering kernel consists of four matrix-valued amplitudes which can be computed within a nonperturbative dressed propagator model $[9,25,27-29]$.

Expressions for the transverse vertex function derived from transverse STIs and constrained by perturbative QCD [30] that satisfy multiplicative renormalizability will be presented elsewhere [51]. A simplified, minimal form of this novel vertex can be expressed by

$$
\begin{aligned}
\Gamma_{\mu}^{\mathrm{STI}}(k, p)= & G\left(q^{2}\right) X_{0}\left(q^{2}\right)\left[\Sigma_{A}\left(k^{2}, p^{2}\right) \gamma_{\mu}\right. \\
& +\Delta_{A}\left(k^{2}, p^{2}\right) \gamma \cdot(k+p)(k+p)_{\mu} \\
& -i \Delta_{B}\left(k^{2}, p^{2}\right)(k+p)_{\mu} \mathbb{I}_{D} \\
& +\frac{\Delta_{A}\left(k^{2}, p^{2}\right)}{2}\left(q^{2} \gamma_{\mu}-\gamma \cdot q q_{\mu}\right) \\
& \left.-\Delta_{B}\left(k^{2}, p^{2}\right) \sigma_{\mu \nu} \gamma_{\nu}\right],
\end{aligned}
$$

where $G\left(q^{2}\right)$ is the ghost-dressing function and $X_{0}\left(q^{2}\right)$ the leading form factor of the quark-ghost kernel $[20,25]$. Additional form factors for arbitrary momenta were obtained in Ref. [28] and can readily be included. For the present purpose their contributions can be neglected and we also set $X_{0}\left(q^{2}\right)=1[20,21]$.

\section{B. Gluon interaction models and lattice QCD dressing functions}

An interaction Ansatz for $\mathcal{G}\left(q^{2}\right)$ that has proven its merits in meson and baryon phenomenology is the MT model [37] and can be decomposed as

$$
\mathcal{G}\left(q^{2}\right)=q^{2} \mathcal{G}_{\mathrm{IR}}\left(q^{2}\right)+4 \pi \tilde{\alpha}_{\mathrm{PT}}\left(q^{2}\right),
$$

where $\tilde{\alpha}_{\mathrm{PT}}\left(q^{2}\right)$ is a monotonically decreasing and regular continuation of the perturbative strong coupling in QCD and $\mathcal{G}_{\mathrm{IR}}\left(q^{2}\right)$ is an Ansatz for the interaction in the infrared domain of squared momenta. $\mathcal{G}_{\mathrm{IR}}\left(q^{2}\right)$ is strongly suppressed for $q^{2} \gtrsim 2 \mathrm{GeV}^{2}$ where $\tilde{\alpha}_{\mathrm{PT}}\left(q^{2}\right)$ dominates.

In all instances we use

$$
4 \pi \tilde{\mathrm{PT}}_{\mathrm{PT}}\left(q^{2}\right)=\frac{8 \pi^{2} q^{2} \gamma_{m} \mathcal{F}\left(q^{2}\right)}{\ln \left[\tau+\left(1+q^{2} / \Lambda_{\mathrm{QCD}}^{2}\right)^{2}\right]},
$$

with $\gamma_{m}=12 /\left(33-2 N_{f}\right), N_{f}=4, \Lambda_{\mathrm{QCD}}=0.234 \mathrm{GeV}, \tau=$ $e^{2}-1$ and $\mathcal{F}\left(q^{2}\right)=\left[1-\exp \left(q^{2} / 4 m_{t}^{2}\right)\right] / q^{2}, m_{t}=0.5 \mathrm{GeV}$. This functional form preserves the one-loop renormalization-group behavior of QCD in the gap equation. The lowmomentum range of the MT model is described by a Gaussian-type support that vanishes in the infrared:

$$
\mathcal{G}_{\mathrm{IR}}^{\mathrm{MT}}\left(q^{2}\right)=\frac{4 \pi^{2}}{\omega^{6}} q^{2} D e^{-q^{2} / \omega^{2}} .
$$

More recently, though, a modified version of this function was proposed [39] which deliberately avoids the $q^{2}$ factor and therefore leads to an infrared massive and finite interaction:

$$
\mathcal{G}_{\mathrm{IR}}^{\mathrm{QC}}\left(q^{2}\right)=\frac{8 \pi^{2}}{\omega^{4}} D e^{-q^{2} / \omega^{2}}
$$

We stress that neither model, Eqs. (18) and (19), in conjunction with Eqs. (16) and (17) can be expressed via a non-negative spectral density. It is also a feature of these interactions that they are virtually insensitive to variations of $0.4 \leq \omega \leq 0.6$ so long as the product $D \omega$ remains constant. It is crucial, though, that the form and parametrization of these models provide enough strength to realize sufficient DCSB. We here use both interaction Ansätze in studying the interplay of effects of CSB and DCSB for different quark flavors in Sec. III.

Along with the vertex Ansatz in Eq. (15) derived from longitudinal and transverse STIs, we make use of Padé approximations [52,53] for unquenched $\left(N_{f}=2+1+1\right)$ lattice-regularized ghost- and gluon-dressing functions, $G^{\text {Latt }}\left(q^{2}\right)$ and $\Delta^{\text {Latt }}\left(q^{2}\right)$, respectively [54]. The integral kernel in Eq. (9) thus becomes

$$
\begin{aligned}
& Z_{1} g^{2} D_{\mu \nu}(q) \Gamma_{\nu}(k, p) \\
& \quad=Z_{2} 4 \pi \alpha_{s} q^{2} \Delta^{\text {Latt }}\left(q^{2}\right) D_{\mu \nu}^{\mathrm{free}}(q) \Gamma_{\nu}^{\mathrm{STI}}(k, p) .
\end{aligned}
$$

\section{The quark sigma term and constituent-quark mass}

A convenient parameter to study the effect of DCSB is the renormalization-point-invariant ratio

$$
\zeta:=\frac{\sigma_{f}}{M_{f}^{E}},
$$


where $\sigma_{f}$ is the constituent-quark sigma term and $M_{f}^{E}$ is the Euclidean constituent mass. In analogy with the nucleon's sigma term, one defines a measure of the contribution from CSB to the constituent-quark mass by $\sigma_{f}:=m_{f}(\mu)\left\langle Q\left|\bar{q}_{f} q_{f}\right| Q\right\rangle$, and using the HellmannFeynman theorem $[55,56]$ this scalar matrix element can be directly related to the constituent-quark mass:

$$
\sigma_{f}=m_{f}(\mu) \frac{\partial M_{f}^{E}}{\partial m_{f}(\mu)},
$$

where $M_{f}^{E}$ is the Euclidean mass function solution [1]:

$$
\left(M_{f}^{E}\right)^{2}:=\left\{p^{2} \mid p^{2}=M^{2}\left(p^{2}\right)\right\} .
$$

Since at the quark level $M_{f}^{E}$ contains both the CSB and DCSB contributions to the quark's mass, the ratio $\zeta$ is zero in the chiral limit and increases with a larger current-quark mass: $0 \leq \zeta \leq 1$. The case $\zeta \simeq 1$ is expected for the topquark mass.

It should be mentioned that the definition of a constituent-quark sigma term independent of its hadronic environment is problematic due to interactions with bystander quarks and chiral corrections are important for light quarks; indeed, the definition of $\sigma_{f}$ ought to depend on the hadron's properties $[57,58]$. Naturally, the effects of DCSB are more comprehensively studied with hadronic $\sigma$ terms, where they measure the contribution of nonvanishing current-quark masses to the nucleon mass.

Here, we merely want to investigate whether different DSE kernels actually affect the relative contributions of DCSB to CSB to the quark mass, as it is well known that the running and functional behavior of the mass function depend on the dressed-vertex Ansatz; our solutions for $M\left(p^{2}\right)$ for all vertex Ansätze confirm this. To this end, the definition of Eq. (21) provides a renormalization-pointindependent measure. In particular, as our results indicate, the charm quark is far from being a heavy quark, and in any of the truncations we consider here, the DCSB contribution to its mass is about $40 \%$. Thus, the frequent use of a constituent charm quark in calculations of charmed masses and form factors is inadequate. Moreover, the relative contributions of CSB and DCSB are fairly independent of the DSE kernel's truncation.

\section{NUMERICAL RESULTS: DCSB AND CSB INTERPLAY AND INTERACTION KERNELS}

The quark-gap equation (1) is numerically solved for the three quark-gluon vertices and dressed interaction models detailed in Secs. II A and II B, where the model parameters $\omega$ and $D$ are chosen such that for a given interaction kernel the experimental light-hadron mass spectrum and weak decay constants are reproduced. We collect them in Table I and use the same parameters for all quark flavors.
TABLE I. Interaction parameters employed in the MT and Qin-Chang (QC) models, Eqs. (18) and (19), respectively, in combination with the three vertex Ansätze described in Sec. II A. In case of the MT model we absorb the renormalization constant $Z_{2}$ in the interaction $\mathcal{G}^{\mathrm{MT}}\left(q^{2}\right)$ but include it in the vertex when using the QC model; see Eq. (9).

\begin{tabular}{lcc}
\hline \hline Model parameters & $(\omega D)^{1 / 3}[\mathrm{GeV}]$ & $\omega[\mathrm{GeV}]$ \\
\hline MT + RL & 0.72 & 0.40 \\
QC + RL & 0.80 & 0.50 \\
MT + BC & 0.65 & 0.50 \\
QC + BC & 0.65 & 0.50 \\
MT + (BC + T $)$ & 0.55 & 0.50 \\
QC + (BC + T $)$ & 0.52 & 0.50 \\
\hline \hline
\end{tabular}

The renormalization conditions (4) and (5) are imposed at $\mu=19 \mathrm{GeV}$ for the vertices in Eqs. (11), (12) and (14) for both the MT and QC interactions. The bare vertex is multiplied by a renormalization constant $Z_{2}$ as in Eq. (11), which introduces a renormalization factor $Z_{2}^{2}$ on the righthand side of Eq. (9). In case of the MT interaction we always absorb $Z_{2}^{2}$ in the interaction function $\mathcal{G}\left(q^{2}\right)$, whereas it is explicitly maintained for the QC interaction; this leads to linear and nonlinear renormalization conditions, respectively [59].

The STI vertex (15) is treated somewhat differently: as indicated in Eq. (20), a linear renormalization condition is imposed but we choose the scale $\mu=4.3 \mathrm{GeV}$ at which the unquenched $\left(N_{f}=2+1+1\right)$ dressing functions $G^{\text {Latt }}\left(q^{2}\right)$ and $\Delta^{\text {Latt }}\left(q^{2}\right)$ were renormalized [54]. Likewise, we employ the light and heavy renormalized quark masses of that same reference: $m_{u, d}(2 \mathrm{GeV}) \simeq 40 \mathrm{MeV}$, $m_{s}(2 \mathrm{GeV})=95 \mathrm{MeV}, m_{c}(2 \mathrm{GeV})=1.51 \mathrm{GeV}$ evolved to the scale $\mu=4.3 \mathrm{GeV}$ and for the beauty quark we choose $m_{b}(4.3 \mathrm{GeV})=M_{b}(4.3 \mathrm{GeV})=4.697 \mathrm{GeV}$ obtained from the solution of the quark DSE (1) with the interaction produced by Eqs. (16), (17) and (19) and the BC + T vertex (14). For comparison, we also solve the same DSE with quenched $\left(N_{f}=0\right)$ and partially quenched $\left(N_{f}=2\right)$ gluon and ghost propagators which are less suppressed than the unquenched ones; however, this is compensated by a decrease of the corresponding value of $\alpha_{s}^{N_{f}}(\mu)$ [54] and the evolution of $\zeta$ in Fig. 4 is fairly independent of $N_{f}$.

In Table II we report the numerical values for the Euclidean constituent-quark masses $M_{f}^{E}$, obtained with the different combinations of interaction and vertex functions that enter the DSE kernel (1). As an illustration of the mass functions, we plot them for four flavors and in the chiral limit in Fig. 2 for the case of solving the DSE with the $\mathrm{BC}+\mathrm{T}$ vertex (14) and the infrared-finite QC interaction (19). The functional behavior is similar for other vertex Ansätze; however, the dressed mass $M\left(p^{2}\right)$ tends to reach its perturbative limit faster in the RL truncation than with the $\mathrm{BC}$ or $\mathrm{BC}+\mathrm{T}$ vertices as a function of $p^{2}$. 
TABLE II. Euclidean quark masses $M_{f}^{E}(\mathrm{GeV})$ as defined in Eq. (23) with the model parameters of Table I. We assume isospin symmetry for the two lightest quarks.

\begin{tabular}{lcccc}
\hline \hline$f$ & $u, d$ & $s$ & $c$ & $b$ \\
\hline$m_{f}(\mu)$ & 0.0037 & 0.082 & 0.970 & 4.100 \\
$\left(M_{f}^{E}\right)^{\mathrm{MT}+\mathrm{RL}}$ & 0.403 & 0.555 & 1.566 & 4.682 \\
$\left(M_{f}^{E}\right)^{\mathrm{QC}+\mathrm{RL}}$ & 0.408 & 0.563 & 1.576 & 4.701 \\
$\left(M_{f}^{E}\right)^{\mathrm{MT}+\mathrm{BC}}$ & 0.385 & 0.512 & 1.505 & 4.648 \\
$\left(M_{f}^{E}\right)^{\mathrm{QC}+\mathrm{BC}}$ & 0.381 & 0.495 & 1.495 & 4.664 \\
$\left(M_{f}^{E}\right)^{\mathrm{MT}+(\mathrm{BC}+\mathrm{T})}$ & 0.387 & 0.533 & 1.549 & 4.693 \\
$\left(M_{f}^{E}\right)^{\mathrm{QC}+(\mathrm{BC}+\mathrm{T})}$ & 0.390 & 0.514 & 1.530 & 4.687 \\
\hline \hline
\end{tabular}

The common and more important feature is the mass function's fast rise and inflection point in the range $1 \mathrm{GeV}^{2} \lesssim p^{2} \lesssim 10 \mathrm{GeV}^{2}$, which can be traced back to the lack of a positive-definite spectral function of the quark propagator and thus confinement $[1,2]$.

It is clear from Table II and Fig. 2 that DCSB plays a substantial role even for the charm quark since it is responsible for nearly $40 \%$ of its constituent mass. Therefore, a careful treatment of heavy-light mesons ought to take this feature into consideration and abandon a constant-mass propagator for the charm [9-14].

The core results of this study are summarized in Table III and Fig. 3, where we depict the evolution of the ratio $\zeta$ as a function of the renormalized current-quark mass for the quark-gluon interaction models listed in Table I. We recover the well-known results for the RL truncation that the constituent-quark mass in case of a light quark is

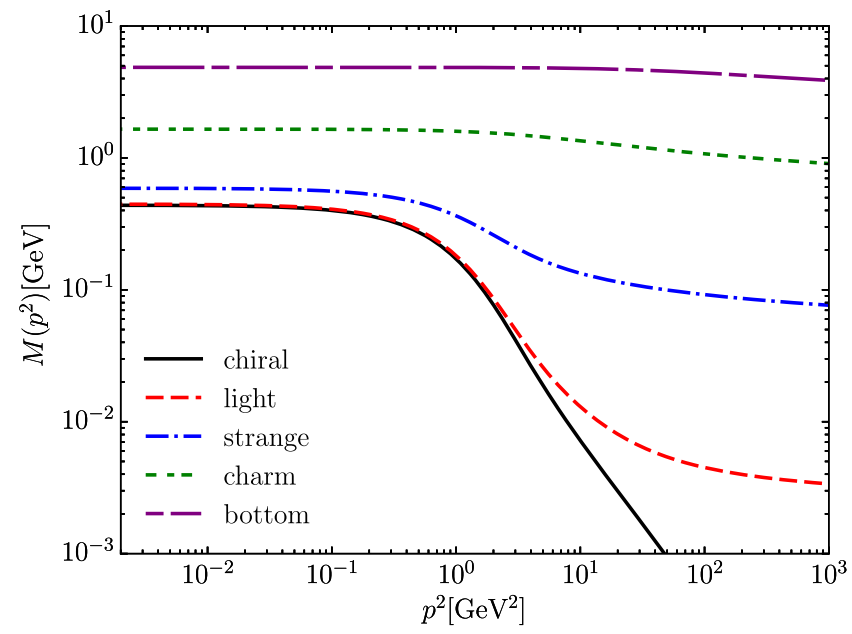

FIG. 2. The running mass $M_{f}\left(p^{2}\right)$ generated by the interaction produced by Eqs. (16), (17) and (19) with $(\omega D)^{1 / 3}=0.52 \mathrm{GeV}$, $\omega=0.50$, and the dressed quark-gluon vertex of Eqs. (12)-(14) with $\eta=0.325$. This leads to $M_{\hat{m}_{f}=0}(0)=0.434 \mathrm{GeV}$ in the chiral limit, $M_{u, d}(0)=0.447 \mathrm{GeV}, M_{s}(0)=0.590 \mathrm{GeV}, M_{c}(0)=$ $1.654 \mathrm{GeV}$ and $M_{b}(0)=4.865 \mathrm{GeV}$.
TABLE III. Flavor dependence of the renormalization-pointinvariant ratio $\zeta(21)$. Models and parameters are as in Table I.

\begin{tabular}{lcccc}
\hline \hline$f$ & $u, d$ & $s$ & $c$ & $b$ \\
\hline$\zeta^{\mathrm{MT}+\mathrm{RL}}$ & 0.025 & 0.234 & 0.642 & 0.851 \\
$\zeta^{\mathrm{QC}+\mathrm{RL}}$ & 0.025 & 0.237 & 0.638 & 0.852 \\
$\zeta^{\mathrm{MT}+\mathrm{BC}}$ & 0.021 & 0.215 & 0.679 & 0.860 \\
$\zeta^{\mathrm{QC}+\mathrm{BC}}$ & 0.018 & 0.216 & 0.691 & 0.864 \\
$\zeta^{\mathrm{MT}+(\mathrm{BC}+\mathrm{T})}$ & 0.019 & 0.235 & 0.665 & 0.850 \\
$\zeta^{\mathrm{QC}+(\mathrm{BC}+\mathrm{T})}$ & 0.019 & 0.224 & 0.678 & 0.852 \\
\hline \hline
\end{tabular}

roughly $98 \%$ due to DCSB, whereas for the $b$ quark it is merely about $15 \%$. Strikingly, it can be inferred from Table III that this observation is nearly independent of the interaction and vertex Ansatz used in the DSE and this is true for all flavors.

One reads from Fig. 3 that $\zeta$ experiences a rapid rise in the range $0.1 \mathrm{GeV} \lesssim m_{f}(\mu) \lesssim 1 \mathrm{GeV}$, that is, in the mass region between $m_{s}(\mu)$ and $m_{c}(\mu)$. Around $m(\mu) \approx 220 \mathrm{MeV}$, an inflection point is followed by a continuing and later attenuated increase of $\zeta$ towards its limiting value. In the RL truncations this increase beyond $0.5 \mathrm{GeV}$ is slightly lower, indicating an enhanced DCSB contribution for heavier quarks compared to the vertices in Eqs. (12) and (14).

As mentioned earlier, we treat the DSE kernel in Eq. (20) separately for the sole reason the lattice-QCD simulations for the ghost and gluon propagators were performed with dynamical light quarks which range from 20 to $50 \mathrm{MeV}$ [54] at $\mu=2 \mathrm{GeV}$. For consistency, we choose $m_{u, d}(2 \mathrm{GeV})=40 \mathrm{MeV}$ evolved to $\mu=4.3 \mathrm{GeV}$, which is where the conditions (4) and (5) are imposed.

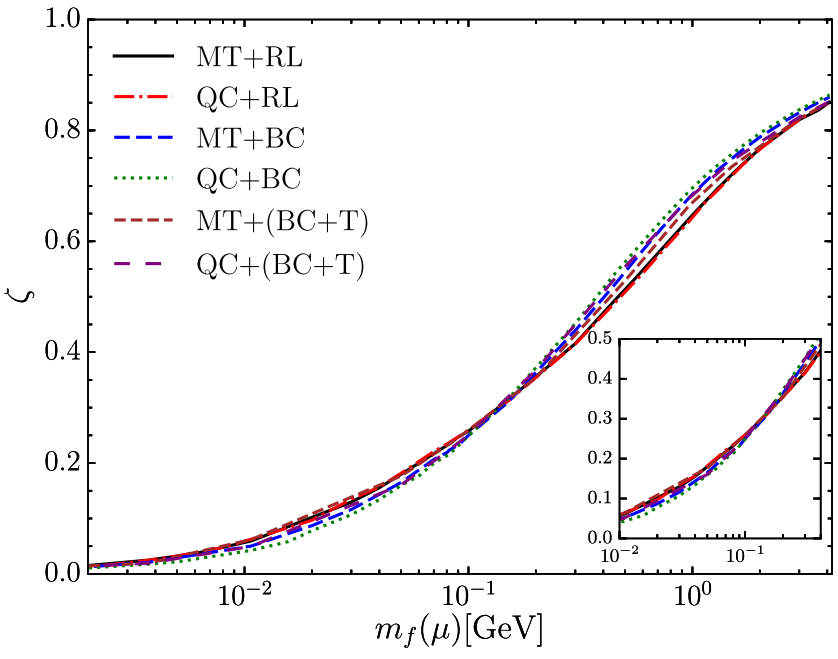

FIG. 3. The renormalization-point-invariant ratio $\zeta$ as a function of $m_{f}(\mu)$ defined in Eq. (21). Models and parameters are as in Table I. 
TABLE IV. Euclidean quark masses $M_{f}^{E}$ and renormalizationpoint-invariant ratio $\zeta$ (21) obtained from the implementation of the integral kernel in Eq. (20). For the solution of the quark DSE we make use of $\alpha_{s}=0.45$ and $\mu=4.3 \mathrm{GeV}$. We also employ $m_{u, d}(\mu)=38.9 \mathrm{MeV}, m_{s}(\mu)=82.1 \mathrm{MeV}, m_{c}(\mu)=1.304 \mathrm{GeV}$ and $m_{b}(\mu)=4.697 \mathrm{GeV}$, which generate $M_{u, d}(0)=0.272 \mathrm{GeV}$, $M_{s}(0)=0.347 \mathrm{GeV}, M_{c}(0)=1.635 \mathrm{GeV}$ and $M_{b}(0)=4.874 \mathrm{GeV}$, respectively.

\begin{tabular}{lcccc}
\hline \hline$f$ & $u, d$ & $s$ & $c$ & $b$ \\
\hline$\left(M_{f}^{E}\right)^{\text {Latt+STI }}$ & 0.257 & 0.324 & 1.547 & 4.671 \\
$\zeta^{\text {Latt+STI }}$ & 0.253 & 0.355 & 0.773 & 0.943 \\
\hline \hline
\end{tabular}

As a consequence, for the light quarks in Table IV the CSB contribution to the constituent-quark mass is more important when compared to the $\zeta$ values in Table III-for light quarks one starts out with $\zeta_{u, d}=0.253$; see Fig. 4 . In other words, the value of the ratio of CSB to the sum of CSB and DCSB is 0.25 . Since the Euclidean constituentquark mass $M_{u, d}^{E}$ is about $36 \%$ lighter with the latticegenerated interaction and the STI vertex (15), it is clear that important tensor structures responsible for DCSB were left out. The complete structure will be discussed elsewhere [51] and produces DCSB in amounts comparable to that exhibited in Table II.

Nevertheless, the chiral symmetry breaking strength of this minimal vertex Ansatz, which does not rely on any model interaction, produces realistic constituent-quark masses for current-quark masses beyond $\sim 100 \mathrm{MeV}$. Therefore, the increase of $\zeta$ in Fig. 4 is initially nearly linear on the logarithmic scale with again a slight inflection point at about $m(\mu) \approx 400 \mathrm{MeV}$, whereas at $m(\mu) \approx$ $220 \mathrm{MeV}$ CSB and DCSB appear to be of equal



FIG. 4. The renormalization-point-invariant ratio $\zeta$ as a function of $m_{f}(\mu)$ obtained with the interaction kernel Eq. (20). importance. The latter observation departs somewhat from what is seen in Fig. 3, where equal contributions occur at $m(\mu) \approx 400 \mathrm{MeV}$.

\section{FINAL REMARKS}

In this work we have investigated for which range of current-quark masses the balance of CSB and DCSB is comparable in dependence of

(i) a chosen nonperturbative gluon-interaction model,

(ii) an Ansatz for the dressed quark-gluon vertex, and

(iii) a DSE kernel based on a minimal vertex from STIs and lattice-gluon and -ghost propagators.

As we have seen, this occurs somewhere midway between the strange and charm mass and is fairly independent of the ingredients in the quark-gap equation. While the gauge-dependent quark-mass function $M\left(p^{2}\right)$ reaches its perturbative limit faster in the RL models considered here than with gauging-technique vertices, the ratio $\zeta$ proves to be largely independent of the details of the integral kernel in the quark DSE.

This, however, is true when the combination of vertex and gluon dressings produces an interaction strength and functional form congruent with that required by hadron phenomenology, i.e. which reproduces the experimental hadron mass spectrum, weak decay constants and electromagnetic form factors.

We went a step further and analyzed the DCSB of a nonperturbative quark-gluon vertex model whose "longitudinal" components constitute a ghost-improved BallChiu vertex [20,25], whereas the transverse vertex consists of relevant tensor structures derived from two transverse STIs [50]. We deliberately neglect contributions from the quark-ghost scattering kernel, as their effect is of minor order than that of the transverse vertex. Solving the DSE with the kernel of Eq. (20) we find massive solutions with considerable DCSB, attenuated for the light and strange quarks and similar to that of phenomenological interactions for the heavy quarks.

This latter DSE kernel does not satisfy the requirements for a sound description of hadron properties, yet crucial improvements are underway [51] and the only inputs are gluon- and ghost-dressing functions for which numerical solutions of DSEs or from lattice QCD are readily available. For the present purposes, this minimal STI vertex suffices to verify that CSB and DCSB are of roughly the same order at a mass scale of about $220 \mathrm{MeV}$, i.e. between the strange- and charm-quark mass.

\section{ACKNOWLEDGMENTS}

B. E. appreciates a stimulating discussion on chiral symmetry breaking with Yu-Xin Liu at Peking University. The authors are grateful to Gastão Krein for insightful comments on the manuscript and to José Rodríguez-Quintero for providing the Padé approximations of the gluon- and ghost-dressing functions. This work was partially supported 
by the Conselho Nacional de Desenvolvimento Científico e Tecnológico (CNPq) Grants No. 168240/2017-3 (F. E. S.) $\begin{array}{llll}\text { and No. 307485/2017-0 } & \text { (B. E.), Coordenação de }\end{array}$ Aperfeiçoamento de Pessoal de Nível Superior (CAPES)
Grant No. 1811288 Edital 086/2013 (F. E. S.) and Fundação de Amparo à Pesquisa do Estado de São Paulo (FAPESP) Grants No. 2016/03154-7 (B. E.) and No. 2015/21550-4 (C. C.).
[1] A. Bashir, L. Chang, I. C. Cloët, B. El-Bennich, Y.X. Liu, C. D. Roberts, and P. C. Tandy, Commun. Theor. Phys. 58, 79 (2012).

[2] I. C. Cloët and C. D. Roberts, Prog. Part. Nucl. Phys. 77, 1 (2014).

[3] G. Eichmann, H. Sanchis-Alepuz, R. Williams, R. Alkofer, and C.S. Fischer, Prog. Part. Nucl. Phys. 91, 1 (2016).

[4] M. A. Ivanov, Y. L. Kalinovsky, and C. D. Roberts, Phys. Rev. D 60, 034018 (1999).

[5] A. Höll, P. Maris, C. D. Roberts, and S. V. Wright, Nucl. Phys. B, Proc. Suppl. 161, 87 (2006).

[6] B. El-Bennich, M. A. Ivanov, and C. D. Roberts, Nucl. Phys. B, Proc. Suppl. 199, 184 (2010).

[7] B. El-Bennich, C. D. Roberts, and M. A. Ivanov, Proc. Sci QCD-TNT-II2012 (2012) 018.

[8] P. Maris and P. C. Tandy, Nucl. Phys. B, Proc. Suppl. 161, 136 (2006).

[9] E. Rojas, B. El-Bennich, and J. P. B. C. de Melo, Phys. Rev. D 90, 074025 (2014).

[10] F. F. Mojica, C. E. Vera, E. Rojas, and B. El-Bennich, Phys. Rev. D 96, 014012 (2017).

[11] B. El-Bennich, EPJ Web Conf. 172, 02005 (2018).

[12] B. El-Bennich, G. Krein, L. Chang, C. D. Roberts, and D. J. Wilson, Phys. Rev. D 85, 031502 (2012).

[13] B. El-Bennich, M. A. Ivanov, and C. D. Roberts, Phys. Rev. C 83, 025205 (2011).

[14] B. El-Bennich, M. A. Paracha, C. D. Roberts, and E. Rojas, Phys. Rev. D 95, 034037 (2017).

[15] A. C. Aguilar, D. Binosi, and J. Papavassiliou, Phys. Rev. D 78, 025010 (2008).

[16] C. S. Fischer, A. Maas, and J. M. Pawlowski, Ann. Phys. (Amsterdam) 324, 2408 (2009).

[17] A. K. Cyrol, M. Mitter, J. M. Pawlowski, and N. Strodthoff, Phys. Rev. D 97, 054006 (2018).

[18] A. I. Davydychev, P. Osland, and L. Saks, Phys. Rev. D 63, 014022 (2000).

[19] R. Alkofer, C. S. Fischer, F. J. Llanes-Estrada, and K. Schwenzer, Ann. Phys. (Amsterdam) 324, 106 (2009).

[20] E. Rojas, J. P. B. C. de Melo, B. El-Bennich, O. Oliveira, and T. Frederico, J. High Energy Phys. 10 (2013) 193.

[21] E. Rojas, B. El-Bennich, J. P. B. C. de Melo, and M. A. Paracha, Few Body Syst. 56, 639 (2015).

[22] S. X. Qin, L. Chang, Y. X. Liu, C. D. Roberts, and S. M. Schmidt, Phys. Lett. B 722, 384 (2013).

[23] D. Binosi, L. Chang, J. Papavassiliou, and C. D. Roberts, Phys. Lett. B 742, 183 (2015).

[24] D. Binosi, L. Chang, J. Papavassiliou, S. X. Qin, and C. D. Roberts, Phys. Rev. D 95, 031501 (2017).
[25] A. C. Aguilar and J. Papavassiliou, Phys. Rev. D 83, 014013 (2011).

[26] A. C. Aguilar, D. Binosi, D. Ibañez, and J. Papavassiliou, Phys. Rev. D 90, 065027 (2014).

[27] A.C. Aguilar, J. C. Cardona, M. N. Ferreira, and J. Papavassiliou, Phys. Rev. D 96, 014029 (2017).

[28] A.C. Aguilar, J. C. Cardona, M. N. Ferreira, and J. Papavassiliou, Phys. Rev. D 98, 014002 (2018).

[29] A. C. Aguilar, M. N. Ferreira, C. T. Figueiredo, and J. Papavassiliou, Phys. Rev. D 99, 034026 (2019).

[30] A. Bashir, R. Bermudez, L. Chang, and C. D. Roberts, Phys. Rev. C 85, 045205 (2012).

[31] R. Bermudez, L. Albino, L. X. Gutiérrez-Guerrero, M. E. Tejeda-Yeomans, and A. Bashir, Phys. Rev. D 95, 034041 (2017).

[32] O. Oliveira, T. Frederico, W. de Paula, and J. P. B. C. de Melo, Eur. Phys. J. C 78, 553 (2018).

[33] J. S. Ball and T. W. Chiu, Phys. Rev. D 22, 2542 (1980).

[34] J. S. Ball and T. W. Chiu, Phys. Rev. D 22, 2550 (1980); 23, 3085(E) (1981).

[35] F. Gao, C. Tang, and Y.x. Liu, Phys. Lett. B 774, 243 (2017).

[36] M. A. Sultan, F. Akram, B. Masud, and K. Raya, arXiv: 1810.01396.

[37] P. Maris and P. C. Tandy, Phys. Rev. C 60, 055214 (1999).

[38] B. El-Bennich, G. Krein, E. Rojas, and F. E. Serna, Few Body Syst. 57, 955 (2016).

[39] S. x. Qin, L. Chang, Y.x. Liu, C. D. Roberts, and D. J. Wilson, Phys. Rev. C 84, 042202 (2011).

[40] P. Maris and C. D. Roberts, Int. J. Mod. Phys. E 12, 297 (2003).

[41] A. Bashir and A. Raya, arXiv:hep-ph/0411310.

[42] J. C. R. Bloch, Phys. Rev. D 66, 034032 (2002).

[43] P. Maris and C.D. Roberts, Phys. Rev. C 56, 3369 (1997).

[44] L. Chang, C. D. Roberts, and S. M. Schmidt, Phys. Rev. C 87, 015203 (2013).

[45] K. I. Kondo, Int. J. Mod. Phys. A 12, 5651 (1997).

[46] M. R. Pennington and R. Williams, J. Phys. G 32, 2219 (2006).

[47] H. X. He, Commun. Theor. Phys. 46, 109 (2006).

[48] A. A. Slavnov, Teor. Mat. Fiz. 10, 153 (1972) [Theor. Math. Phys. 10, 99 (1972)].

[49] J. C. Taylor, Nucl. Phys. B33, 436 (1971).

[50] H. x. He, Phys. Rev. D 80, 016004 (2009).

[51] L. Albino, A. Bashir, L. X. Gutiérrez-Guerrero, B. El-Bennich, and E. Rojas, arXiv:1812.02280.

[52] A. Bashir, A. Raya, and J. Rodríguez-Quintero, Phys. Rev. D 88, 054003 (2013). 
[53] D. Binosi, C. D. Roberts, and J. Rodríguez-Quintero, Phys. Rev. D 95, 114009 (2017).

[54] A. Ayala, A. Bashir, D. Binosi, M. Cristoforetti, and J. Rodríguez-Quintero, Phys. Rev. D 86, 074512 (2012).

[55] H. Hellmann, Z. Phys. 85, 180 (1933).

[56] R. P. Feynman, Phys. Rev. 56, 340 (1939).
[57] A. W. Thomas and G. Krein, Phys. Lett. B 481, 21 (2000).

[58] D. B. Leinweber, S. Boinepalli, I. C. Cloet, A. W. Thomas, A. G. Williams, R. D. Young, J. M. Zanotti, and J. B. Zhang, Phys. Rev. Lett. 94, 212001 (2005).

[59] T. Hilger, M. Gómez-Rocha, A. Krassnigg, and W. Lucha, Eur. Phys. J. A 53, 213 (2017). 